\title{
ANALYsis OF The SATURATION ThroughPUT IN AN IEEE 802.11 NeTWORK USING COGNITIVE RADIO
}

\author{
Jun Peng \\ Electrical Engineering Department, University of Texas - Rio Grande Valley \\ Edinburg, TX 78539 USA
}

\begin{abstract}
We present a Markov chain model to estimate the saturation throughput of an IEEE 802.11 network in the basic access mode using cognitive radio. IEEE 802.11 networks are very popular and likely more spectrum is needed for their future deployment and applications. On the other hand the cognitive radio idea has been proposed to deal with the spectrum shortage problem. It is thus reasonable to assume that future IEEE 802.11 network may operate in the context of cognitive radio. One paradigm is that IEEE 802.11 networks will act as secondary users sharing the spectrum of a primary use using the dynamic spectrum access approach. In this scenario it will be important to study how the physical layer of cognitive radio may impact on the IEEE 802.11 network as a whole. Based on a Markov chain model, we investigate the impact of the primary user's arrivals on the IEEE 802.11 network's saturation throughput. We validate our presented model with ns 2 simulations. As shown by our numerical results, our model results are very close to the $n$ s 2 simulation results.
\end{abstract}

\section{KEYWORDS}

Saturation throughput, IEEE 802.11, network protocols, medium access control

\section{INTRODUCTION}

Cognitive radio (CR) was proposed to improve the efficiency of using spectrum without disrupting the operations of existing spectrum owners. To address the spectrum shortage challenge posed by the ubiquitous demands for high-speed wireless networks of the society, the spectrum regulatory bodies, such as the Federal Communications Commission (FCC), and the industrial and research communities have been investigating ways to use the scarce spectrum more efficiently [1].

One promising approach is the dynamic spectrum access (DSA) based on cognitive radio [1]. An ideal cognitive radio device can sense its spectral environment, analyze the environment, reason for best responses, and finally adapt to the environment by adopting new operating parameters. Besides, spectrum usage analysis found that many owners of spectrum do not always heavily use their spectrum during some hours or in some areas [1], which opens the door for DSA.

The basic idea of DSA is to allow secondary users adopting cognitive radios to share the spectrum with a primary user or users, which are the spectrum's owner . Equipped with cognitive radios, the secondary users can determine if the primary user is using the spectrum. When the primary user is using the spectrum, the secondary users will back off. However, when the primary

DOI: 10.5121/ijwmn.2017.9504 
user is not using the spectrum, the secondary users can send data through the spectrum. Obviously, when the primary user becomes active again, the secondary users need to stop their transmission yielding to the primary user.

There are two paradigms envisioned for cognitive radio networks [1]. One is that the second users in a cognitive radio network have their own infrastructure, while the other is that they are fully distributed. In this paper we focus on the IEEE 802.11's distributed coordination function which is for distributed networks. Without loss of generality, we assume a single primary user in the rest of the text.

The question is how the cognitive radio physical layer would affect the upper layers and the cognitive radio network as whole. We are interested in answering this question for the popular IEEE 802.11 networks in its basic mode. Particularly, we present an analysis on the saturated throughput of the IEEE 802.11 in its basic mode using cognitive radio.

One popular Markov chain model on the IEEE 802.11 operation was proposed by Bianchi [2] and later revised by many other researchers. In the original model the channel of the IEEE 802.11 network has no losses except when there is collision in the network. To analyze an IEEE 802.11 network having a lossy channel, Dong et al. [3] and Ni et al. [4] revised the model to accommodate channel losses not caused by collision. Both papers assume that the lossy channel has a constant bit error rate.

In an IEEE 802.11 network using cognitive radio the primary user can become active at any given time, which causes frame loss in the secondary network of IEEE 802.11. The loss distribution will be much different from that assumed in $[3,4]$ and the proposed models in $[3,4]$ will thus be invalid. To address this new scenario, we propose a revised Markov chain model incorporating the signal arrivals of the primary user.

The rest of our paper is organized as follows. Section II introduces the basic concepts used in our model and saturation throughput analysis. Section III presents the Markov chain model. Section IV analyzes the saturation throughput of an IEEE 802.11 network in the basic mode using cognitive radio. Section $\mathrm{V}$ shows simulation results validating the proposed model. Finally, Section VI concludes our paper.

\section{AbStracting The IeEe 802.11 Network In Medium Time}

We need to abstract the IEEE 802.11 network from the primary user's network for its analysis, since their traffic are interleaved in the medium. Only when the primary user is not using the spectrum can IEEE 802.11 nodes use the spectrum as secondary users. When the primary user starts to use the spectrum again, the IEEE 802.11 nodes must yield and stop transmitting into the spectrum. The IEEE 802.11 network is thus an overlay opportunistic network over the primary user's network.

Although the medium times of the IEEE 802.11 network and the primary user's network are interleaved, we can separate the medium times of the two networks since in general these two networks are not active in the medium at the same time. Specifically, we can erase the primary user's times in the time domain and join the rest of the time segments together, which forms the 
medium time picture of the IEEE 802.11 network. This abstracting process is the basis of our analysis.

In the abstracted IEEE 802.11 network, we apply another concept introduced in [3,5], which is virtual slot. As defined in [3], a virtual slot is basically the time interval between two consecutive backoff counter decrements of the nodes in the network that are not transmitting. A backoff counter in IEEE 802.11 can decrements only after the channel has been idle for a specified amount of time. Virtual slots present the channel activities and can therefore be used to build a Markov chain model on the operation of the IEEE 802.11 network [3]. We apply the concept of virtual slot in our abstracted IEEE 802.11 network.

The activities of the IEEE 802.11 nodes define the virtual slots in the channel. The IEEE 802.11 standard defines two coordination functions. One is the point coordination function (PCF) for infrastructure based networks, while the other is the distributed coordination function (DCF) that is for infrastructure-less networks. Further, the IEEE 802.11 distributed coordination function defines a basic access mechanism that uses two-way handshaking and another optional access mechanism using four-way handshaking.

The IEEE 802.11 distributed coordination function is based on CSMA/CA $[6,7,8,9]$ in which a node must listen to the channel before a transmission to determine if it can start the transmission. An IEEE 802.11 node will not abort an on-going transmission because it does not transmit and listen at the same time in general. An IEEE 802.11 node equipped with cognitive radio is expected to keep on monitoring the spectrum using various detectors, such as energy detector, feature detector, and matched filter detector. However, it's very difficult for a node to detect an arriving signal when itself is transmitting in the same channel, even when the node has more than one antennas, since its own transmitting antenna and receiving antenna would be very close to each other as compared with the node's distance to a primary user. The detector that has the highest probability for a successful detection in such as case would be a matched filter, but it is also the most challenging detector to implement in a real scenario [1].

Another proposed method for primary user detection is cooperative sensing which requires the secondary users or even third party nodes to cooperate in detecting the primary user's signal. The primary user's signal is geographically diverse due to the complexity of wireless propagation and thus it needs further investigation on how the results of the primary user detectors can be shared among the secondary users. In addition, when a secondary user is transmitting it is either impossible to deliver the primary user status to it or it will be very costly, such as using a separate control channel, which would be against the original spectrum efficiency goal of cognitive radio.

Moreover, even if the primary user status is updated through a control channel, the secondary user transmitting a frame has most likely already finished its transmission when it receives the primary user status update. First, primary user detectors have delays in detecting the arrival of primary user's signal [1]. Second, the detectors' outputs need to be collected, processed, and "merged" per specific algorithms. Third, the status frame has to undergo the same various delays of a normal frame before it reaches a secondary user, such as transmission, propagation, and queuing delays. We thus can reasonably assume that a secondary user node finishes transmitting a frame after it starts its transmission.

The IEEE 802.11 distributed coordination function defines two access methods. One is the the basic access method, while the other is the optional RTS/CTS access method [10]. We focus on 
the basic access method in this paper. The basic access method uses a two-way handshaking mechanism. Before a node transmits a frame, it first senses the medium for other nodes' radio activities. If the medium is sensed idle without transmission for a duration more than the Distributed Inter-Frame Space (DIFS), the node can proceed to transmit the frame.

However, if the node senses the medium busy, it defers until it senses an idle duration more than DIFS and then starts a random back-off timer. The node draws the timer's value uniformly from a Contention Window $(\mathrm{CW})$ that it maintains. The back-off timer keeps decreasing as long as the node senses the medium idle. The timer pauses when the node senses the medium busy, but the timer is resumed for decreasing when the node senses the medium idle for more than DIFS again. When the timer expires by reaching zero, the node transmits the data frame. If the receiver receives the frame successfully, it sends back an Acknowledge (ACK) frame after waiting for a duration of Short Inter-Frame Space (SIFS). When either the data or ACK frame is not successfully received, the data frame will be retransmitted and the contention window will be doubled. Besides, other nodes hearing the data frame will reserve the medium for the pair of nodes by adjusting their Network Allocation Vector (NAV), a technique known as virtual carrier sensing.

An IEEE 802.11 distributed coordination function cycle in the basic access method thus has the sequence of "DATA-ACK". The cycle, however, may terminate early for many reasons. In our case of cognitive radio networks having a lossless channel, the distributed coordination function cycle may terminate early for two reasons. One is that there is a collision among the secondary user nodes (i.e., the IEEE 802.11 nodes), while the other is that the primary user's signal arrives during the distributed coordination function cycle.

There are thus four types of virtual slots in the secondary user network: 1 . an idle virtual slot; 2. a virtual slot of a corrupted data frame or frames; 3. a virtual slot of a corrupted ACK frame; and 4. a virtual slot of a successful distributed coordination function cycle. The durations of these four virtual slots in the abstracted secondary user network are

$$
\begin{gathered}
T_{1}=\delta \\
T_{2}=\Gamma+\sigma+\mathrm{EIFS}+\delta \\
T_{3}=\Gamma+\sigma+\mathrm{SIFS}+\mathrm{ACK}+\sigma+\mathrm{EIFS}+\delta \\
T_{4}=\Gamma+\sigma+\mathrm{SIFS}+\mathrm{ACK}+\sigma+\mathrm{DIFS}+\delta
\end{gathered}
$$

where ACK is the transmission duration of the ACK frame, DIFS, SIFS and EIFS are the interframe spaces, $\delta$ is the slot time defined by the physical layer, $\sigma$ is the link propagation time, and $\Gamma$ is the transmission time of a data frame. As in [3], transmissions are separated by at least one idle slot used by the non-transmission nodes as a back off slot.

\section{The Markov Chain Model}

The Markov chain model shows the state transitions in the secondary user network of distributed coordination function nodes. The minimum contention window is $W$ in the secondary user 
network, and the maximum number of back off stages in the network is $m$ for each node, where $W, m \in N_{0}$. At backoff stage $i$, where $i \in[0, m]$ and $i \in N_{0}$, the contention window $W_{i}$ is given by

$$
W_{i}=2^{i} W
$$

The initial backoff counter value $C_{i} \in N_{0}$ at stage $i$ for each node is uniformly chosen in $\left[0, W_{i}-1\right]$.

Same as in [3], the stochastic process $\{s(n), b(n)\}$ is modeled as the Markov chain shown in Fig. 1 , where $n$ is the virtual slot number, $s(n)$ is the backoff stage number, and $b(n)$ is the backoff counter. Note that in this model the maximum backoff counter value at stage $i$ is $W_{i}-2$ instead of $W_{i}-1$ because each virtual slot includes one backoff slot for non-transmitting nodes [3].

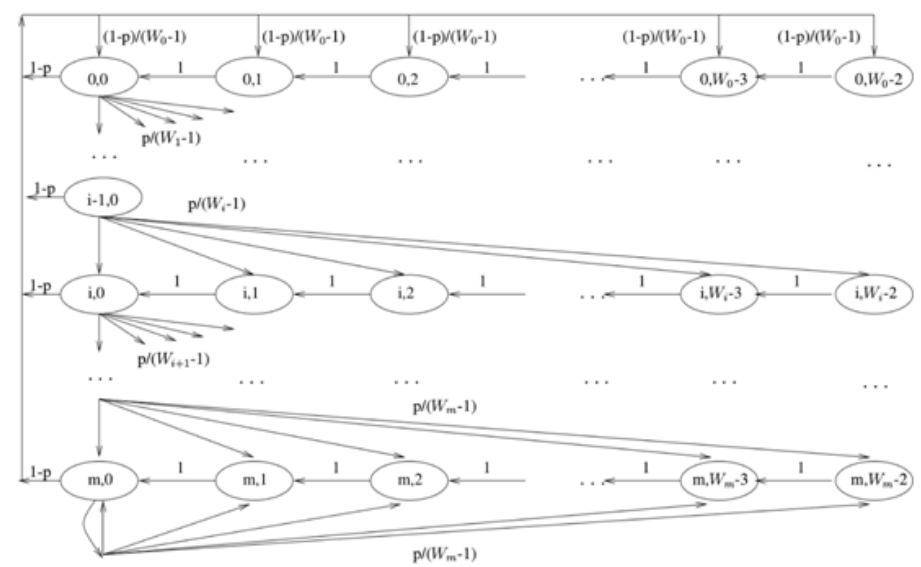

Figure 1: The Markov Chain model

The model assumes that there are $n$ nodes in the secondary user network where the nodes hear each other. Besides, there is no capture in reception for each node in the network. Let $p$ be the probability that the distributed coordination function's DATA-ACK terminates without completion in a virtual cycle. When the channel is lossless, the distributed coordination function cycle may terminate for two reasons, as mentioned earlier, a collision in the secondary user network or the arrival of the primary user's signal. Let $\tau$ be the probability that a node transmits in a virtual slot.

As in the models presented in [2,3], it is assumed that $p$ and $\tau$ are constant and virtual slots are independent from each other. The one-step transition probabilities in the Markov chain are as follows $\left(k \in N_{0}\right)$ :

$$
\begin{gathered}
P\{i, k \mid i, k+1\}=1 \quad k \in\left[0, W_{i}-3\right], i \in[0, m] \\
P\{0, k \mid i, 0\}=\frac{1-p}{W_{0}-1} k \in\left[0, W_{0}-2\right], i \in[0, m]
\end{gathered}
$$




$$
\begin{aligned}
& P\{i, k \mid i-1,0\}=\frac{p}{W_{i}-1} k \in\left[0, W_{i}-2\right], i \in[1, m] \\
& P\{m, k \mid m, 0\}=\frac{p}{W_{m}-1} k \in\left[0, W_{m}-2\right]
\end{aligned}
$$

Although the transitions in the model shown in Fig. 1 show the same medium activities as in the models presented in [2, 3], the $p$ is different from those defined in [2,3]. As defined earlier, $p$ represents the probability that the distributed coordination function's DATA-ACK cycle terminates early in a virtual slot, either due to a collision in the secondary user network or the arrival of primary user's signal.

The probability of collision for a frame in the secondary user network, $P_{c}$, depends on $\tau$, the probability of a node's transmission in a slot. When one or more of the $(n-1)$ secondary user nodes start to transmit in the virtual slot of a frame, there is a collision on the frame. We therefore have

$$
P_{c}=1-(1-\tau)^{(n-1)}
$$

At the same time, the probability that the primary user's signal arrives during a distributed coordination function's cycle, $P_{a}$, depends on the primary user's traffic intensity. We may assume that the primary user has a Poisson traffic arrival with an average rate of $\lambda$. We thus then have

$$
P_{a}=1-e^{-\lambda . T_{c}}, \text { where } T_{c}=\Gamma+\sigma+\mathrm{SIFS}+\mathrm{ACK}+\sigma .
$$

Note that $\sigma$ is the propagation delay, while $\Gamma$ is the transmission duration of a data frame. We can also reasonably assume that the collision among the secondary user nodes is independent of the primary user's arrivals. Therefore,

$$
p=P_{c}+P_{a}-P_{c} \cdot P_{a}
$$

From these equations, we can derive

$$
\tau=1-\left(\frac{1-p}{1-p_{a}}\right)^{1 /(n-1)}
$$

In addition, after solving the balance equations in the Markov chain as in $[2,3]$, we can obtain the stationary distribution of the Markov chain, $b_{i, k}$. The stationary distribution can be used to derive $\tau$ in terms of $p, W$, and $m$. The result is shown as below. 


$$
\tau=\sum_{i=0}^{m} b_{i, 0}=\frac{2(1-2 p)}{(1-2 p) W+p(W-1)\left(1-(2 p)^{m}\right)}
$$

The unique values of $\tau$ and $p$ are determined by the two equations 12 and 13.

As stated in [2], the time in this model is stopped when the channel is sensed busy, which means that the Markov chain is basically driven by idle slots. When the primary user is active in the medium, the state machine of the secondary user network is "paused" staying in the state where the primary user happens to become active. This further shows that in analyzing the secondary user network we can abstract the secondary user network from the primary user's activities.

\section{The Saturation ThroughPUT}

We define the saturation throughput of the secondary user network based on the time segments during which the secondary user nodes are active. When the secondary user nodes are yielding to the primary user, the medium time should not be counted for the secondary user network. In other words, our throughput analysis is on the abstracted secondary user network.

The normalized saturation throughput of the secondary user network, $S$, is defined as:

$$
S=\frac{E\left(T_{p l d}\right)}{E\left(T_{s u}\right)}
$$

where $T_{p l d}$ is the medium time used to transmit the MAC payload in the secondary user network and $T_{s u}$ is the medium time available for the secondary user network.

The amount of time that the primary user stays active each time after it becomes active does not affect our throughput analysis on the secondary user network. However, the distribution of the primary user's traffic arrivals is one key factor in determining the transition probabilities in the Markov chain, which is modeled as a Poisson process with an average arrival rate of $\lambda$. In the rest of our analysis we focus on the abstracted secondary user network.

Let $P_{i d l e}$ and $P_{t r}$ be the probabilities of no node transmitting in a virtual slot and of at least one node transmitting in a virtual slot, respectively.

$$
\begin{gathered}
P_{i d l e}=(1-\tau)^{n} \\
P_{t r}=1-P_{i d l e}=1-(1-\tau)^{n}
\end{gathered}
$$

Let $P_{S}$ be the probability that one and only one node transmits in a virtual slot, conditioned on that there is at least one node transmitting.

$$
P_{s}=\frac{{ }_{n} C_{1} \tau(1-\tau)^{(n-1)}}{1-(1-\tau)^{n}}=\frac{n \tau(1-\tau)^{(n-1)}}{1-(1-\tau)^{n}}
$$


Let $P_{S S}$ be the probability that there is exactly one node transmitting and during the DATA frame transmission the primary user does not become active, conditioned on that there is at least one node transmitting.

$$
P_{s s}=P_{s} e^{-\lambda(\Gamma+\sigma)}
$$

We now can calculate the probabilities of the virtual slots in the secondary user network. We defined four types of virtual slots and their probabilities are as follows, respectively:

$$
\begin{gathered}
P_{1}=P_{i d l e} e^{-\lambda . \delta} \\
P_{2}=P_{t r}\left(1-P_{s s}\right) \\
P_{3}=P_{t r} P_{s s}\left(1-e^{-\lambda(\mathrm{SIFS}+\mathrm{ACK}+\sigma)}\right) \\
P_{4}=P_{t r} P_{s s} e^{-\lambda(\mathrm{SIFS}+\mathrm{ACK}+\sigma)}
\end{gathered}
$$

Note that a frame can be corrupted by the primary user's signal when any of its bits is in transmission or propagation, which explains the $\sigma$ in the formulas.

The saturation throughput $S$ is then

$$
S=\frac{P_{4} L}{\sum_{i=1}^{4} P_{i} T_{i}},
$$

where $L$ is the transmission time of the MAC payload in a DATA frame. 


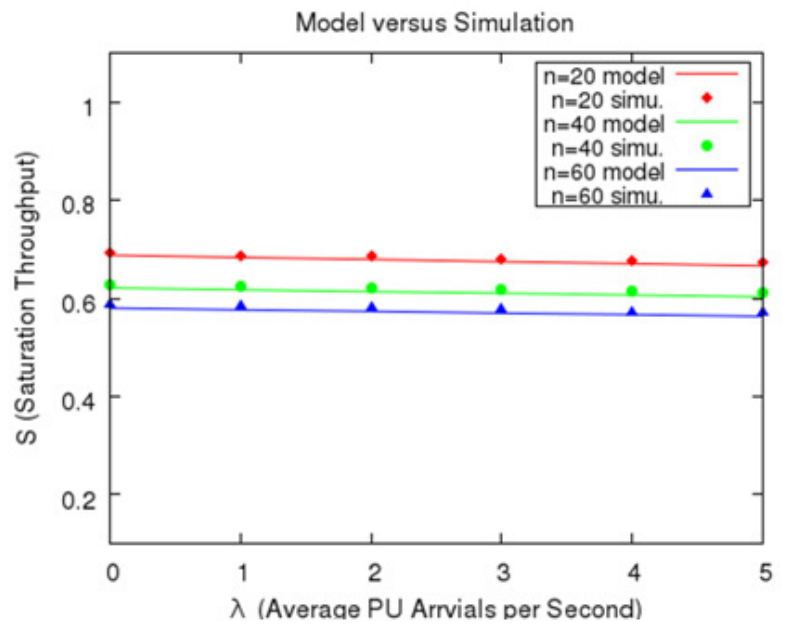

Figure 2: Model Results versus Simulation Results

\section{MOdel VALidation}

We validate the model with the ns2 simulator [11]. As in [3], the physical layer is chosen to approximate the IEEE 802.11 Direct Sequence Spread Spectrum (DSSS) radio with a speed of 1 $\mathrm{Mb} / \mathrm{s}$ and the short and long retry limits are 255. The constant MAC payload is 8000 bits per frame. The propagation delay, $\sigma$, in the model is assumed to be 1 microsecond. In both simulation and analysis we have saturation traffic at each node.

We vary the average rate of the primary user's arrivals, $\lambda$, in the simulations to study how the primary user impacts on the saturation throughput of the secondary user network. Specifically, $\lambda$ varies from 0 to 5 arrivals per second. Fig. 2 shows three cases in which the number of secondary user nodes is 20,40, and 60, respectively. As shown in the figure, the analysis results match the simulation results closely in all tested cases.

\section{Conclusions}

Based on the abstracted network idea in the context of cognitive radio networks, we revised a Markov chain model on IEEE 802.11 throughput to address the new scenario of IEEE 802.11 using cognitive radio. With a lossless channel, a frame transmitted by an IEEE 802.11 node, a secondary user, may be corrupted in two cases. In the first case the frame collides with another frame transmitted by another IEEE 802.11 node. In the second case the frame is corrupted by the primary user's signal. The original model proposed by Bianchi deals with the collision losses, while some revised models address a lossy channel. Our revised model deals with frame losses caused by both frame collision inside the secondary user network and the arrival of the primary user's signal. Based on the revised model, we derived the saturation throughput of an IEEE 802.11 network using cognitive radios. We also used simulations to validate our throughput analysis. The simulation results match closely with our analysis results in all the cases we tested. 


\section{REFERENCES}

[1] B. Wang and K. J. R. Liu, "Advances in cognitive radio networks: A survey," IEEE Journal of Selected Topics in Signal Processing, vol. 5, no. 1, pp. 5-23, Feb 2011.

[2] G. Bianchi, "IEEE 802.11-saturation throughput analysis," IEEE Communications Letters, vol. 2, no. 12, pp. 318-320, Dec 1998.

[3] X. J. Dong and P. Varaiya, "Saturation throughput analysis of IEEE 802.11 wireless LANs for a lossy channel," IEEE Communications Letters, vol. 9, no. 2, pp. 100-102, Feb 2005.

[4] Q. Ni, T. Li, T. Turletti, and Y. Xiao, "Saturation throughput analysis of error-prone 802.11 wireless networks: Research articles,” Wirel. Commun. Mob. Comput., vol. 5, no. 8, pp. 945-956, Dec. 2005.

[5] V. Vishnevsky and A. Lyakhov, "802.11 LANs: Saturation Throughput in the Presence of Noise," in Second International IFIP-TC6 Networking Conference Pisa, Italy, May 19-24, 2002 Proceedings, 2002.

[6] A. Colvin, "CSMA with collision avoidance," Computer Commun., vol. 6, pp. 227-235, 1983.

[7] P. Karn, "MACA - a new channel access method for packet radio," in Proc. of the 9th ARRL Computer Networking Conference, Ontario, Canada, 1990.

[8] C. L. Fullmer and J. J. Garcia-Luna-Aceves, "Solutions to hidden terminal problems in wireless networks," in Proc. of the ACM SIGCOMM, French Riviera, France, September 1997.

[9] V. Bharghavan, A. Demers, S. Shenker, and L. Zhang, "MACAW: a medium access protocol for wireless LANs," in Proc. of the ACM SIGCOMM, London, United Kingdom, August 1994.

[10] IEEE 802.11 wireless local area networks. [Online]. Available: http://grouper.ieee.org/groups/802/11/

[11] The network simulator - ns-2. [Online]. Available: http://www.isi.edu/nsnam/ns/

\section{Authors}

Jun Peng received his Ph.D. degree from the Department of Electrical, Computer \& Systems Engineering at Rensselaer Polytechnic Institute, Troy, New York, United States. He is currently an associate professor at the Electrical Engineering Department of University of Texas - Rio Grande Valley, Edinburg, Texas, United States. His research interests are in computer and communication networks. 